\title{
Solution of Lane-Emden Type Equations by Combination of the Spectral Method and Adomian Decomposition Method
}

\author{
S. Gh. Hosseini and S. Abbasbandy \\ Department of Mathematics, Science and Research Branch, Islamic Azad University, Tehran, Iran \\ Correspondence should be addressed to S. Gh. Hosseini; ghasem602@yahoo.com
}

Received 26 December 2014; Revised 1 April 2015; Accepted 6 April 2015

Academic Editor: Maria Gandarias

Copyright (C) 2015 S. Gh. Hosseini and S. Abbasbandy. This is an open access article distributed under the Creative Commons Attribution License, which permits unrestricted use, distribution, and reproduction in any medium, provided the original work is properly cited.

\begin{abstract}
The application of a new modified Adomian decomposition method for obtaining the analytic solution of Lane-Emden type equations is investigated. The proposed method, called the spectral Adomian decomposition method, is based on a combination of spectral method and Adomian decomposition method. A comparative study between the proposed method and Adomian decomposition method is presented. The obtained result reveals that method is of higher efficiency, validity, and accuracy.
\end{abstract}

\section{Introduction}

Recently, the studies on singular initial value problems (IVPs) for second order ordinary differential equations (ODEs) have been the focus of considerable attention. One of the second order equations describing this type of problem is the LaneEmden singular IVPs, which can be written in the form of

$$
y^{\prime \prime}+\frac{\alpha}{x} y^{\prime}+f(y)=g(x) \quad \alpha \geq 0,0 \leq x \leq \xi,
$$

subject to initial conditions:

$$
\begin{array}{r}
y(0)=A, \\
y^{\prime}(0)=B,
\end{array}
$$

where $A$ and $B$ are constants, $f(y)$ is a continuous real valued function, and $g(x) \in C[0,1]$. The Lane-Emden equation was first studied by two astrophysicists, Jonathan Homer Lane and Robert Emden, who examined the thermal behavior of a spherical cloud of gas acting under the mutual attraction of its molecules subject to the classical laws of thermodynamics [1]. The well-known Lane-Emden equation has been applied to model several phenomena in mathematical physics and astrophysics such as theory of stellar structure, the thermal behavior of a spherical cloud of gas, isothermal gas spheres, and the theory of thermionic currents [2]. Approximate solutions to the abovementioned problems were presented by Wazwaz $[3,4]$ by applying the Adomian decomposition method which provides a convergent series solution. Nouh [5] accelerated the convergence of a power series solution of the Lane-Emden equation by using an Euler-Able transformation and pade approximation. Exact solution of generalized Lane-Emden solutions of the first kind is investigated by Goenner and Havas [6]. Liao [7] solved Lane-Emden type equations by applying a homotopy analysis method. He [8] obtained an approximate analytical solution of the Lane-Emden equation by applying a variational approach which uses a semi-inverse method. Yousefi [9] converted the Lane-Emden equation to an integral equation and then used Legendre wavelets and achieved an approximate solution for $0<x \leq 1$. Momoniat and Harley [10] applied the Lie Group method successfully to the generalized Lane-Emden equation of the first kind. The solution of a class of singular second order IVPs of Lane-Emden type was presented by Yildirim and Öziş [11] and also Chowdhury and Hashim [12] by He's homotopy perturbation method. Ramos [13] provided a series approach to the Lane-Emden equation and compared it with He's homotopy perturbation method. The exact solutions of this equation (for some cases) were provided by Khalique and Ntsime [14] through Noether point symmetry approach. Singh et al. [15] applied the modified homotopy analysis method for solving this equation for cases $f(y)=y^{n}$ and 
$f(y)=\left(y^{2}-c\right)^{3 / 2}$. Parand et al. gained numerical solutions by using Rational Legendre Pseudospectral approach [16]. The variational iteration method for Lane-Emden singular type equations was employed by Yıldırım and Öziş [17]. Iqbal and Javed [18] presented a new powerful semianalytic technique by optimal homotopy asymptotic method and obtained numerical solutions for some cases. Numerical solution for this equation was presented by Pandey and Kumar [19] where they applied Bernstein operational matrix of differentiation. Bhrawy and Alofi [20] established numerical solutions for some types of Lane-Emden equation through Jacobi-Gauss collocation method. Heydari et al. [21] employed radial basis functions and used integral operator to solve this equation for some various types. A new shifted second kind chebyshev operational matrix of derivatives was introduced by Doha et al. [22] where they reduced the Lane-Emden type equations to a system of algebraic equations and gained numerical results for a variety of cases. Nazari-Golshan et al. [23] performed a modified homotopy perturbation method coupled with the Fourier transform and solved three different singular nonlinear Lane-Emden equations.

In exploring the analytic solution of Lane-Emden equations by $\mathrm{ADM}$, some calculation problems may occur. In this work we incorporate the spectral method and ADM to overcome these difficulties. By using this method, the time consumption will be reduced, and under some conditions the spectral Adomian decomposition method can be proved to be convergent. In this paper the hybrid spectral Adomian decomposition method will be described in Section 2, while in Section 3, the convergence of ADM and SADM will be presented. The test problem will be interpreted and also the obtained results will be compared by some others method in Section 4, and finally in Section 5, the conclusion in details is provided.

\section{Hybrid Spectral Adomian Decomposition Method}

2.1. Adomian Decomposition Method for Lane-Emden Equation. The Adomian decomposition method (ADM) is a semianalytical method for ordinary and partial nonlinear differential equations. The details of this method are presented by Adomian [24]. The ADM presented the equation in an operator form by considering the highest-ordered of derivative in the problem. However, a slight change is necessary to overcome the singularity behavior at $x=0$. Hence, in this problem we choose the differential operator $\mathscr{L}$ in terms of the two derivatives, $y^{\prime \prime}+(\alpha / x) y^{\prime}$; (1) can be rewritten in the following form:

$$
\mathscr{L} y=-f(y)+g(x)
$$

where the differential operator $\mathscr{L}$ is

$$
\mathscr{L}=x^{-\alpha} \frac{d}{d x}\left(x^{\alpha} \frac{d}{d x}\right) .
$$

The inverse operator $\mathscr{L}^{-1}$ is

$$
\mathscr{L}^{-1}(\cdot)=\int_{0}^{x} x^{-\alpha} \int_{0}^{x} x^{\alpha}(\cdot) d x d x .
$$

Operating with $\mathscr{L}^{-1}$ on (3), it follows

$$
y(x)=A+B x+\mathscr{L}^{-1} g(x)-\mathscr{L}^{-1} f(y) .
$$

For the solution $y(x)$, the ADM introduces an infinite series

$$
y(x)=\sum_{n=0}^{\infty} y_{n}(x)
$$

and the infinite series of polynomials

$$
f(y)=f\left(\sum_{n=0}^{\infty} y_{n}(x)\right)=\sum_{n=0}^{\infty} A_{n}\left(y_{0}, y_{1}, \ldots, y_{n}\right),
$$

for the nonlinear term $f(y)$, where $A_{n}$, the Adomian polynomials, are obtained as follows:

$$
A_{n}=\frac{1}{n_{!}} \frac{d^{n}}{d \lambda^{n}}\left[f\left(\sum_{i=0}^{n} \lambda^{i} y_{i}\right)\right]_{\lambda=0}, \quad n=0,1,2, \ldots .
$$

By setting (7) and (8) in (6),

$$
\sum_{n=0}^{\infty} y_{n}(x)=A+B x+\mathscr{L}^{-1} g(x)-\mathscr{L}^{-1}\left(\sum_{n=0}^{\infty} A_{n}\right)
$$

will be obtained. To specify the components $y_{n}(x), \mathrm{ADM}$ which indicates the use of recursive relation will be applied:

$$
\begin{aligned}
y_{0}(x) & =A+B x+\mathscr{L}^{-1}(g(x)), \\
y_{k+1}(x) & =-\mathscr{L}^{-1}\left(A_{k}\right), \quad k \geq 0,
\end{aligned}
$$

which gives

$$
\begin{aligned}
& y_{0}(x)=A+B x+\mathscr{L}^{-1}(g(x))=A+B x \\
& \quad+\int_{0}^{x} t^{-\alpha}\left(\int_{0}^{t} s^{\alpha} g(s) d s\right) d t, \\
& y_{1}(x)=-\mathscr{L}^{-1}\left(A_{0}\right)=-\int_{0}^{x} t^{-\alpha}\left(\int_{0}^{t} s^{\alpha} A_{0}(s) d s\right) d t, \\
& y_{2}(x)=-\mathscr{L}^{-1}\left(A_{1}\right)=-\int_{0}^{x} t^{-\alpha}\left(\int_{0}^{t} s^{\alpha} A_{1}(s) d s\right) d t, \\
& \quad \vdots \\
& y_{k+1}(x)=-\mathscr{L}^{-1}\left(A_{k}\right) \\
& =-\int_{0}^{x} t^{-\alpha}\left(\int_{0}^{t} s^{\alpha} A_{k}(s) d s\right) d t .
\end{aligned}
$$

The series solution is $y(x)=y_{0}(x)+y_{1}(x)+y_{2}(x)+\cdots$ and the $n$-term approximation of the series solution will be denoted as $\phi_{n}(x)=\sum_{i=0}^{n-1} y_{i}(x)$. This method has been used to solve different equations; see $[24,25]$. 
2.2. Chebyshev Polynomials. Chebyshev polynomials of the first kind are orthogonal with respect to the weight function $\omega(x)=1 / \sqrt{1-x^{2}}$ on the interval $[-1,1]$ and satisfy the following recursive formula:

$$
\begin{aligned}
T_{0}(x) & =1, \\
T_{1}(x) & =x \\
T_{n+1}(x) & =2 x T_{n}(x)-T_{n-1}(x), \quad n=1,2, \ldots
\end{aligned}
$$

This system is orthogonal basis with weight function $\omega(t)=$ $\left(1-x^{2}\right)^{-1 / 2}$ and orthogonality property:

$$
\int_{-1}^{1} T_{n}(x) T_{m}(x)\left(1-x^{2}\right)^{-1 / 2} d x=\frac{\pi}{2} c_{n} \delta_{n m}
$$

where $c_{0}=2, c_{n}=1$ for $n \geq 1$ and $\delta_{n m}$ is the Kronecker delta function.

A function $u(x) \in L_{\omega}^{2}(-1,1)$ can be expanded by Chebyshev polynomial as follows:

$$
u(x)=\sum_{j=0}^{\infty} u_{j} T_{j}(x)
$$

where the coefficients $u_{j}$ are

$$
u_{j}=\frac{2}{\pi}\left\langle u(x), T_{j}(x)\right\rangle_{\omega}, \quad j=0,1,2, \ldots
$$

Here, $\langle\cdot, \cdot\rangle_{\omega}$ is the inner product of $L_{\omega}^{2}(-1,1)$. The grid (interpolation) points are chosen to be the extrema

$$
x_{i}=-\cos \left(\frac{i \pi}{m}\right), \quad i=0,1, \ldots, m,
$$

of the $m$ th order Chebyshev polynomials $T_{m}(x)$. The following approximation of the function $u(x)$ can be introduced:

$$
u(x) \simeq u^{[m]}(x)=\sum_{j=0}^{m} \widetilde{u}_{j} T_{j}(x)
$$

where $\tilde{u}_{j}$ are the Chebyshev coefficients. These coefficients are determined as follows:

$$
\tilde{u}_{j}=\frac{2(-1)^{j}}{m \widetilde{c}_{j}} \sum_{i=0}^{m} \frac{1}{\widetilde{c}_{i}} u\left(x_{i}\right) \cos \left(\frac{\pi i j}{m}\right), \quad j=0,1, \ldots, m,
$$

where

$$
\widetilde{c}_{i}= \begin{cases}2 & i=0, m \\ 1 & 1 \leq i \leq m-1\end{cases}
$$

The Chebyshev polynomials are widely used for numerical solutions of differential, integral, and integrodifferential equations; see [21, 22].
2.3. The Methodology. At first, based on initial condition (2), the initial approximation $y_{0}(x)=A+B x+\mathscr{L}^{-1}(g(x))$ is selected. By applying iteration formula (12), the following will be obtained:

$$
y_{1}(x)=-\mathscr{L}^{-1}\left(A_{0}\right) .
$$

From (18), the function $y_{1}(x)$ on $[0, \xi]$ can be approximated as follows:

$$
y_{1}(x) \simeq y_{1}^{[m]}(x)=\sum_{j=0}^{m} \tilde{y}_{1 j} T_{j}\left(\frac{2}{\xi} x-1\right),
$$

where $\tilde{y}_{1 j}$ are the Chebyshev coefficients which are determined from (19) as follows:

$$
\begin{aligned}
& \tilde{y}_{1 j}=\frac{2(-1)^{j}}{m \widetilde{c}_{j}} \sum_{i=0}^{m} \frac{1}{\widetilde{c}_{i}} y_{1}\left(\tilde{x}_{i}\right) \cdot \cos \left(\frac{\pi i j}{m}\right), \\
& \quad j=0,1, \ldots, m, \\
& \tilde{x}_{i}=\frac{\xi}{2}\left(x_{i}+1\right), \quad i=0, \ldots, m .
\end{aligned}
$$

For finding the unknown coefficient $y_{1}\left(\tilde{x}_{i}\right), i=0,1, \ldots, m$, by substituting the grid points $\tilde{x}_{i}, i=0,1, \ldots, m$ in (21), the following will be concluded:

$$
y_{1}\left(\tilde{x}_{i}\right)=-\mathscr{L}^{-1}\left(A_{0}\left(\tilde{x}_{i}\right)\right) .
$$

From (23) and (24),

$$
\begin{array}{r}
\tilde{y}_{1 j}(x)=\frac{2(-1)^{j}}{m \widetilde{c}_{j}} \sum_{i=0}^{m} \frac{-1}{\widetilde{c}_{i}} \mathscr{L}^{-1}\left(A_{0}\left(\widetilde{x}_{i}\right)\right) \cdot \cos \left(\frac{\pi i j}{m}\right), \\
j=0,1, \ldots, m
\end{array}
$$

can be gained. Therefore, from (22) and (25) the approximation of $y_{1}(x)$ can be obtained.

For finding the approximation of $y_{2}(x)$, from (12) the following will be gained:

$$
y_{2}(x)=-\mathscr{L}^{-1}\left(A_{1}\right) .
$$

In a similar way, the function $y_{2}(x)$ on $[0, \xi]$ can be approximated as

$$
y_{2}(x) \simeq y_{2}^{[m]}(x)=\sum_{j=0}^{m} \tilde{y}_{2 j} T_{j}\left(\frac{2}{\xi} x-1\right),
$$

where

$$
\tilde{y}_{2 j}=\frac{2(-1)^{j}}{m \widetilde{c}_{j}} \sum_{i=0}^{m} \frac{1}{\widetilde{c}_{i}} y_{2}\left(\tilde{x}_{i}\right) \cdot \cos \left(\frac{\pi i j}{m}\right),
$$

$$
j=0,1, \ldots, m \text {. }
$$

Similarly, for finding the unknown coefficient $y_{2}\left(\tilde{x}_{i}\right), i=$ $0,1, \ldots, m$, by substituting the grid points $\tilde{x}_{i}, i=0,1, \ldots, m$ in (21),

$$
y_{2}\left(\tilde{x}_{i}\right)=-\mathscr{L}^{-1}\left(A_{1}\left(\tilde{x}_{i}\right)\right)
$$


can be concluded; therefore, from (28) and (29)

$$
\begin{array}{r}
\tilde{y}_{2 j}=\frac{2(-1)^{j}}{m \widetilde{c}_{j}} \sum_{i=0}^{m} \frac{-1}{\widetilde{c}_{i}} \mathscr{L}^{-1}\left(A_{1}\left(\tilde{x}_{i}\right)\right) \cdot \cos \left(\frac{\pi i j}{m}\right), \\
j=0,1, \ldots, m
\end{array}
$$

will be gained. From (27) and (30) the approximation of $y_{2}(x)$ can be obtained.

Generally, for $n \geq 2$, according to the above method, the approximation of $y_{n}(x)$ will be achieved as follows:

$$
y_{n}(x) \simeq y_{n}^{[m]}(x)=\sum_{j=0}^{m} \tilde{y}_{n j} T_{j}\left(\frac{2}{\xi} x-1\right),
$$

where

$$
\begin{aligned}
\tilde{y}_{n j}=\frac{2(-1)^{j}}{m \widetilde{c}_{j}} \sum_{i=0}^{m} \frac{-1}{\widetilde{c}_{i}} \mathscr{L}^{-1}\left(A_{n-1}\left(\tilde{x}_{i}\right)\right) \cdot \cos \left(\frac{\pi i j}{m}\right), \\
j=0,1, \ldots, m .
\end{aligned}
$$

At the end, $y_{0}^{[m]}(x)+y_{1}^{[m]}(x)+y_{2}^{[m]}(x)+\cdots+y_{n}^{[m]}(x)$ is the $(n, m)$ term approximation of the series solution.

\section{Convergence}

3.1. Convergence of ADM. According to [26], (12) can be rewritten as follows:

$$
y_{k+1}(x)=\int_{0}^{x}(t-x) x^{\alpha} t^{-\alpha} A_{k}(t) d t .
$$

If $F(x, t)=(t-x) x^{\alpha} t^{-\alpha}$ then

$$
y_{k+1}(x)=\int_{0}^{x} F(x, t) A_{k}(t) d t .
$$

Let the function $f$ in (3) satisfy a Lipschitz condition with Lipschitz constant $L$, and $U$ is an upper bound for the above function $F$; that is, $|f(z)-f(y)| \leq L|z-y|$, and $|F(x, t)| \leq U$, $0 \leq t \leq x \leq \xi$; consequently the following theorem can be achieved.

Theorem 1. The series solution (7) of problem (1) applying $A D M$ converges if $0<\lambda<1$ and $\left|y_{1}\right|<\infty$, where $\lambda=L U \xi$; see [27].

Theorem 2. The maximum absolute truncation error of series solution (7) to problem (1) is estimated to be $\max _{\forall x \in J} \mid y(x)-$ $\sum_{i=0}^{n} y_{i}(x) \mid \leq K \lambda^{n+1} / L(1-\lambda)$ where $K=\max _{\forall x \in J}|f(y(x))|$ and $J=[0, \xi]$; see [27].

\subsection{Convergence of Spectral Method}

Theorem 3. Let $u(t) \in H^{k}(-1,1)$ (Sobolev space) and $u^{[m]}(t)=\sum_{j=0}^{m} \tilde{u}_{j} T_{j}(t) ;$ then

$$
\left\|u(t)-u^{[m]}(t)\right\|_{L_{\omega}^{2}[-1,1]} \leq C_{0} m^{-k}\|u(t)\|_{H_{\omega}^{k}(-1,1)},
$$

where $C_{0}$ is a positive constant, which depends on a selected norm and is independent of $u(t)$ and $m$; see [28].
3.3. Convergence of SADM. Since $y_{\mathrm{ADM}}$, the series solution of problem (1) using ADM for $n$-terms, is

$$
y_{\mathrm{ADM}}(x)=\sum_{i=0}^{n} y_{i}(x),
$$

and also $y_{\mathrm{SADM}}$, the series solution of problem (1) using $\operatorname{SADM}$ for $(n, m)$-terms, is

$$
y_{\mathrm{SADM}}(x)=\sum_{i=0}^{n} y_{i}^{[m]}(x),
$$

SADM can be shown to be convergent by the following theorem.

Theorem 4. The series solution (37) of problem (1) using $S A D M$ converges if $0<\lambda<1,\left|y_{1}^{[m]}\right|<\infty$, and $n \leq m$.

Proof. Consider $y_{\text {exact }}$ to be the exact solution of (1); then

$$
\begin{aligned}
& \left|y_{\text {exact }}(x)-y_{\mathrm{SADM}}(x)\right| \\
& =\left|y_{\text {exact }}(x)-y_{\mathrm{ADM}}(x)+y_{\mathrm{ADM}}(x)-y_{\mathrm{SADM}}(x)\right| \\
& \leq\left|y_{\text {exact }}(x)-y_{\mathrm{ADM}}(x)\right| \\
& \quad+\left|y_{\mathrm{ADM}}(x)-y_{\mathrm{SADM}}(x)\right| .
\end{aligned}
$$

According to Theorem 1,

$$
\left|y_{\text {exact }}(x)-y_{\mathrm{ADM}}(x)\right| \leq \frac{K \lambda^{n+1}}{L(1-\lambda)} .
$$

Also, according to Theorem $3,\left|y_{i}-y_{i}^{[m]}\right| \leq C_{0} m^{-k}\left\|y_{i}(x)\right\|$; Therefore,

$$
\begin{aligned}
& \left|y_{\mathrm{ADM}}(x)-y_{\mathrm{SADM}}(x)\right| \\
& \quad=\sum_{i=0}^{n}\left|y_{i}-y_{i}^{[m]}\right| \leq \frac{n C_{0}}{m^{k}} G \leq \frac{C_{0}}{m^{k-1}} G,
\end{aligned}
$$

where $G=\max _{1 \leq i \leq n}\left\|y_{i}(x)\right\|$. From (39) and (40) the result will be

$$
\left|y_{\text {exact }}(x)-y_{\text {SADM }}(x)\right| \leq \frac{K \lambda^{n+1}}{L(1-\lambda)}+\frac{C_{0}}{m^{k-1}} G .
$$

Thus, with the increase in $n$ and $m, y_{\mathrm{SADM}}$ is convergent to $y_{\text {exact }}$.

\section{Test Problems}

Example 1 (see $[3,19])$. Isothermal gas spheres equation:

$$
y^{\prime \prime}+\frac{2}{x} y^{\prime}+e^{y}=0
$$

with initial conditions

$$
\begin{gathered}
y(0)=0, \\
y^{\prime}(0)=0 .
\end{gathered}
$$


TABLE 1: Comparison of the numerical result of Example 1 by present method $(n=12$ and $m=20)$ and numerical values [3, 19].

\begin{tabular}{lcccccc}
\hline$x$ & BOM [19] & RK4 & SADM & ADM [3] & Error (SADM) & Error (ADM) \\
\hline 0 & 0 & 0 & 0 & 0 & 0 & 0 \\
0.1 & -0.0016658338 & -0.0016658338 & -0.0016658339 & -0.0016658339 & $9.37 e-11$ & $9.5 e-11$ \\
0.2 & -0.0066533671 & -0.0066533659 & -0.0066533671 & -0.0066533671 & $9.37 e-9$ & $8.94 e-10$ \\
0.3 & $*$ & -0.0149328824 & -0.0149328833 & -0.0149328833 & $8.2 e-9$ \\
0.4 & $*$ & -0.0264554772 & -0.0264554763 & -0.0264554763 & $8.78 e-10$ & $9.2 e-10$ \\
0.5 & -0.0411541005 & -0.0411539557 & -0.0411539573 & -0.0411539568 & $1.57 e-9$ & $1.1 e-9$ \\
0.6 & $*$ & -0.0589440694 & -0.0589440748 & -0.0589440720 & $5.4 e-9$ & $2.7 e-9$ \\
0.7 & $*$ & -0.0797260141 & -0.0797260042 & -0.0797599229 & $9.83 e-9$ & $2.2 e-8$ \\
0.8 & $*$ & -0.1033860526 & -0.1033860532 & -0.1033860110 & $6.2 e-10$ & $4.2 e-8$ \\
0.9 & $*$ & -0.1297985460 & -0.1297985243 & -0.1297983987 & $2.17 e-8$ & $1.5 e-7$ \\
1 & -0.1588624338 & -0.1588276877 & -0.1588276775 & -0.1588273537 & $1.02 e-8$ & $3.3 e-7$ \\
\hline
\end{tabular}

TABLE 2: Comparison of the numerical result of Example 2, (Case 1), by present method $(n=12$ and $m=40)$ and numerical values [19].

\begin{tabular}{|c|c|c|c|c|c|c|}
\hline$x$ & BOM [19] & SADM & ADM [3] & Error (BOM) & Error (SADM) & Error (ADM) \\
\hline 0 & 1 & 1 & 1 & 0 & 0 & 0 \\
\hline 0.1 & 0.99833582 & 0.99833583 & 0.99833583 & $7.27 e-10$ & $2.25 e-10$ & $3.3 e-9$ \\
\hline 0.2 & $*$ & 0.99337309 & 0.99337332 & $*$ & $2.64 e-10$ & $2.3 e-7$ \\
\hline 0.3 & $*$ & 0.98519979 & 0.98520236 & $*$ & $1.41 e-9$ & $2.6 e-6$ \\
\hline 0.4 & * & 0.97395826 & 0.97397252 & * & $4.38 e-9$ & $1.4 e-5$ \\
\hline 0.5 & 0.95983907 & 0.95983907 & 0.95989274 & $4.42 e-8$ & $7.32 e-9$ & $5.4 e-5$ \\
\hline 0.6 & $*$ & 0.94307317 & 0.94323076 & $*$ & $7.77 e-9$ & $1.6 e-4$ \\
\hline 0.7 & $*$ & 0.92392284 & 0.92431254 & $*$ & $1.1 e-8$ & $3.4 e-4$ \\
\hline 0.8 & $*$ & 0.90267209 & 0.90352147 & $*$ & $2 e-8$ & $8.5 e-4$ \\
\hline 0.9 & * & 0.78961717 & 0.88129745 & * & $2.71 e-8$ & $1.7 e-3$ \\
\hline 1 & 0.85505757 & 0.85505757 & 0.85813584 & $3.74 e-5$ & $2.73 e-8$ & $3 e-3$ \\
\hline
\end{tabular}

A series solution obtained by Wazwaz [3] using ADM is $y(x)=(-1 / 6) x^{2}+(1 / 120) x^{4}+(-1 / 1890) x^{6}+(61 /$ $1632960) x^{8}+(-629 / 224532000) x^{10}+O(11)$. This example is solved by the SADM with $n=12$ and $m=20$. The numerical results are shown in Table 1 , and it is compared with the ADM [3] and Bernstein operational matrix (BOM) [19].

Example 2 (see $[3,19])$. Consider the following Lane-Emden equation:

$$
y^{\prime \prime}+\frac{2}{x} y^{\prime}+y^{k}=0,
$$

with initial conditions

$$
\begin{gathered}
y(0)=1, \\
y^{\prime}(0)=0,
\end{gathered}
$$

where $k \geq 0$.

Case 1 (for $k=3$ ). A series solution obtained by Wazwaz [3] using ADM is $y(x)=1-(1 / 6) x^{2}+(1 / 40) x^{4}-(1 / 5040) x^{6}+$ $(1 / 1088640) x^{8}+(1 / 598752000) x^{10}+O(11)$. This example is solved by the SADM with $n=12$ and $m=40$. The numerical results are shown in Table 2, and it is compared with the BOM [19]. The residual error is illustrated in Figure 1(a), which proves the proposed method is of high accuracy.
Case $2($ for $k=5)$. The exact solution is $y(x)=\left(1+x^{2} / 3\right)^{-1 / 2}$. A series solution obtained by Wazwaz [3] using ADM is $y(x)=1-(1 / 6) x^{2}+(1 / 24) x^{4}-(1 / 3024) x^{6}+(1 / 653184) x^{8}+$ $(1 / 359251200) x^{10}+O(11)$. This example is solved by the SADM with $n=14$ and $m=20$. The numerical results are shown in Table 3, while the absolute error is illustrated in Figure 2 and it is compared with absolute error expressed by BOM [19] which shows that the proposed method is more accurate.

Example 3 (see [3]). Consider the following Lane-Emden equation:

$$
y^{\prime \prime}+\frac{2}{x} y^{\prime}+\sin (y)=0
$$

with initial conditions

$$
\begin{gathered}
y(0)=1, \\
y^{\prime}(0)=0 .
\end{gathered}
$$

A series solution obtained by Wazwaz [3] using ADM is $y(x)=1-(1 / 6) k_{1} x^{2}+(1 / 120) k_{1} k_{2} x^{4}+k_{1}\left((1 / 3024) k_{1}^{2}-(1 /\right.$ $\left.5040) k_{2}^{2}\right) x^{6}+k_{1} k_{2}\left((-113 / 3265920) k_{1}^{2}+(1 / 362880) k_{2}^{2}\right) x^{8}+$ $k_{1}\left((1781 / 898128000) k_{1}^{2} k_{1}^{2}-(1 / 39916800) k_{2}^{4}-(19 /\right.$ $\left.23950080) k_{1}^{4}\right) x^{10}+O(11)$, where $k_{1}=\sin (1)$ and $k_{2}=\cos (1)$. 
TABLE 3: The numerical results for Example 2, Case 2, for $n=14$ and $m=20$.

\begin{tabular}{lccccc}
\hline$x$ & SADM & Exact & ADM [3] & Absolute error (SADM) & Absolute error (ADM) \\
\hline 0 & 1 & 1 & 1 & 0 & 0 \\
0.1 & 0.998337489 & 0.998337489 & 0.99833750 & $3.4 e-17$ & $1.2 e-8$ \\
0.2 & 0.993399268 & 0.993399268 & 0.99339998 & $4.8 e-17$ & $7.1 e-7$ \\
0.3 & 0.985329278 & 0.985329278 & 0.98533726 & $1.8 e-16$ & $8 e-6$ \\
0.4 & 0.974354704 & 0.974354704 & 0.97439865 & $1.7 e-16$ & $4.4 e-5$ \\
0.5 & 0.960768923 & 0.960768923 & 0.96093234 & $8.75 e-18$ & $1.6 e-4$ \\
0.6 & 0.944911183 & 0.944911183 & 0.94538460 & $2.19 e-15$ & $4.7 e-4$ \\
0.7 & 0.927145541 & 0.927145541 & 0.92829868 & $1.96 e-13$ & $1.1 e-3$ \\
0.8 & 0.907841299 & 0.907841299 & 0.91031357 & $1.03 e-11$ & $2.5 e-3$ \\
0.9 & 0.887356510 & 0.887356509 & 0.89216242 & $3.38 e-10$ & $4.8 e-3$ \\
1 & 0.866025411 & 0.866025404 & 0.87467085 & $7.61 e-9$ & $8.6 e-3$ \\
\hline
\end{tabular}

TABLE 4: Comparison of the numerical result of Example 3 by present method ( $n=11$ and $m=80)$ and ADM [3].

\begin{tabular}{|c|c|c|c|c|c|}
\hline$x$ & RK4 & SADM & ADM [3] & Error (SADM) & Error (ADM) \\
\hline 0.1 & 0.99859793 & 0.99859793 & 0.99859793 & $6.22 e-11$ & $1 e-10$ \\
\hline 0.2 & 0.99439626 & 0.99439626 & 0.99439626 & $3.49 e-11$ & 0 \\
\hline 0.5 & 0.96517781 & 0.96517778 & 0.96517778 & $5.62 e-10$ & $2 e-10$ \\
\hline 0.8 & 0.91183204 & 0.91183203 & 0.91183204 & $4.6 e-9$ & $6.6 e-8$ \\
\hline 1.1 & 0.83609012 & 0.83609015 & 0.83609012 & $3.01 e-8$ & $1.7 e-6$ \\
\hline 1.3 & 0.77443518 & 0.77443523 & 0.77443518 & $4.31 e-8$ & $8.8 e-6$ \\
\hline 1.5 & 0.70504518 & 0.70504523 & 0.70504518 & $5.69 e-8$ & $3.6 e-5$ \\
\hline 1.6 & 0.66783443 & 0.66783449 & 0.66783443 & $6.38 e-8$ & $6.8 e-5$ \\
\hline 1.7 & 0.62917734 & 0.62917741 & 0.62917734 & $6.96 e-8$ & $1.2 e-4$ \\
\hline 1.8 & 0.58926208 & 0.58926216 & 0.58926208 & $7.22 e-8$ & $2.1 e-4$ \\
\hline 1.9 & 0.54828781 & 0.54828789 & 0.54828781 & $7.92 e-8$ & $3.6 e-4$ \\
\hline 2 & 0.50646355 & 0.50646362 & 0.50646355 & $7.57-8$ & $6 e-4$ \\
\hline
\end{tabular}

This example is solved by the SADM with $n=11$ and $m=80$ on $[0,2]$. The numerical results are demonstrated in Table 4, and it is compared with the ADM [3]; the residual error is illustrated in Figure 1(b), which proves the proposed method is of high accuracy.

Example 4. Consider the following Lane-Emden equation:

$$
y^{\prime \prime}+\frac{2}{x} y^{\prime}+\left(8 e^{y}+4 e^{y / 2}\right)=0
$$

with initial conditions

$$
\begin{gathered}
y(0)=0, \\
y^{\prime}(0)=0 .
\end{gathered}
$$

This example is solved by the SADM with $n=10$ and $m=40$. The numerical results are shown in Table 5 , and the absolute errors are illustrated in Figure 3. The exact solution is $y(x)=-2 \ln \left(1+x^{2}\right)$; see [14].

Example 5 (see [3]). Consider the following Lane-Emden equation:

$$
y^{\prime \prime}+\frac{2}{x} y^{\prime}+(-6 y-4 y \ln (y))=0
$$

with initial conditions

$$
\begin{gathered}
y(0)=1, \\
y^{\prime}(0)=0 .
\end{gathered}
$$

A series solution obtained by Wazwaz [3] using ADM is $y(x)=1+x^{2}+(1 / 6) x^{6}+(1 / 24) x^{8}+(1 / 120) x^{10}+O(11)$. This example is solved by the SADM with $n=14$ and $m=40$. The numerical results are shown in Table 6 , and the absolute error is illustrated in Figure 3. The exact solution is $y(x)=e^{x^{2}}$; see [14].

Example 6 (see [3]). Consider the following Lane-Emden equation:

$$
y^{\prime \prime}+\frac{2}{x} y^{\prime}+\left(y^{2}-C\right)^{3 / 2}=0
$$

with initial conditions

$$
\begin{gathered}
y(0)=1, \\
y^{\prime}(0)=0 .
\end{gathered}
$$

A series solution obtained by Wazwaz [3] using ADM is $y(x)=1-(1 / 6) q^{3} x^{2}+(1 / 40) q^{4} x^{4}-(1 / 7 !) q^{5}\left(5 q^{2}+14\right) x^{6}$ 
TABLE 5: The numerical results for Example 4 for $n=10$ and $m=40$.

\begin{tabular}{lccc}
\hline$x$ & SADM & Exact & Absolute error \\
\hline 0.1 & -0.01990066 & -0.01990066 & $1.80200000 e-23$ \\
0.2 & -0.07844143 & -0.07844143 & $7.35634177 e-17$ \\
0.3 & -0.17235539 & -0.17235539 & $5.27102199 e-13$ \\
0.4 & -0.29684001 & -0.29684001 & $2.78978334 e-10$ \\
0.5 & -0.44628707 & -0.44628710 & $3.52756402 e-8$ \\
0.6 & -0.61496760 & -0.61496940 & 0.00000180 \\
0.7 & -0.79750315 & -0.79755224 & 0.00004904 \\
0.8 & -0.98854610 & -0.98939248 & 0.00084639 \\
\hline
\end{tabular}

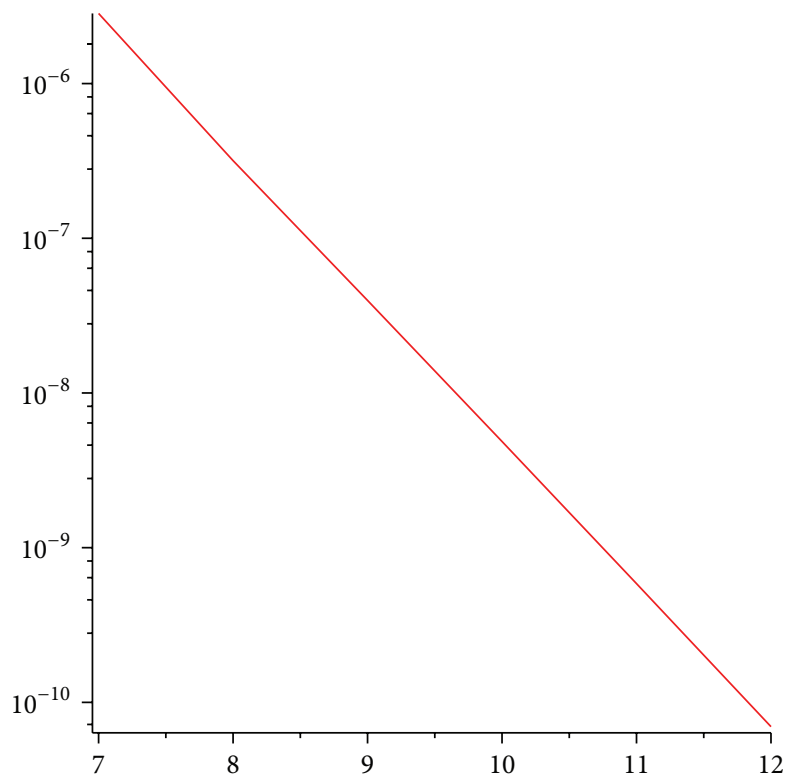

(a)

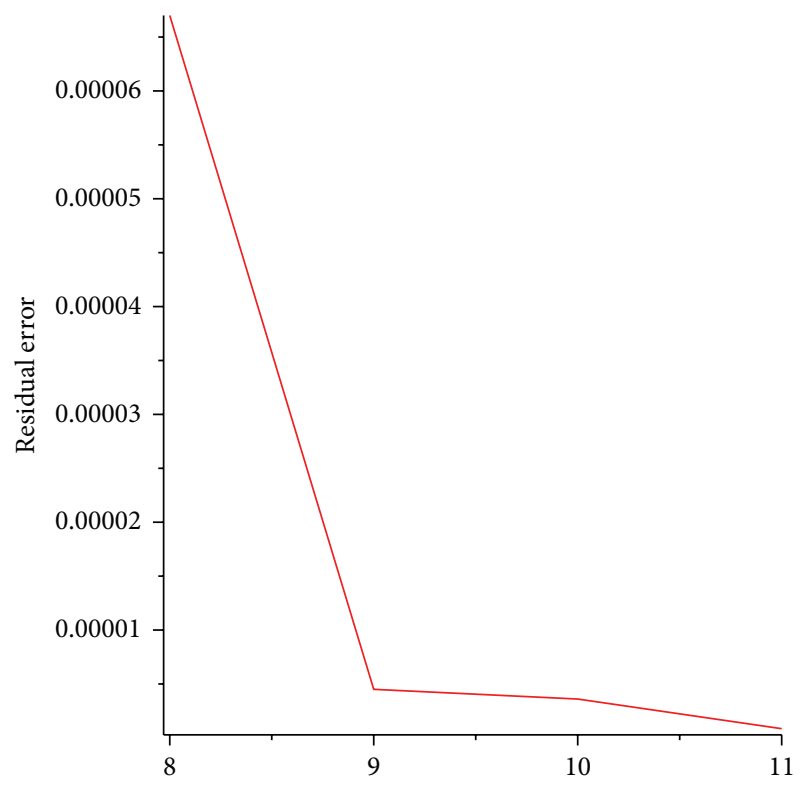

(b)

Figure 1: (a) Example 2, Case 1: Horizontal axis is related number of iteration $(n)$ and vertical axis shows residual error in mod logarithmic. (b) Example 3: Horizontal axis is related number of iteration $(n)$ and vertical axis shows residual error.

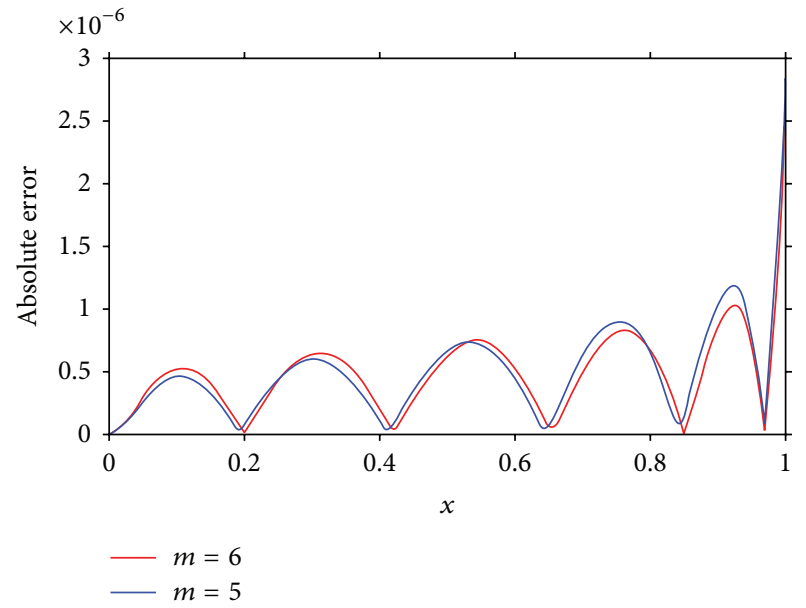

(a)

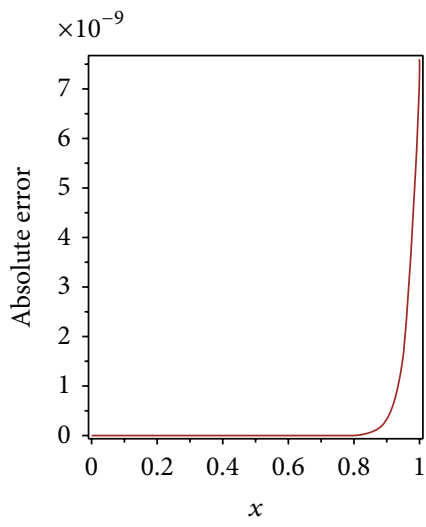

(b)

Figure 2: Absolute error for Example 2, Case 2. (a) Absolute error obtained by SADM $(n=14, m=20)$. (b) Absolute error obtained by BOM [19]. 


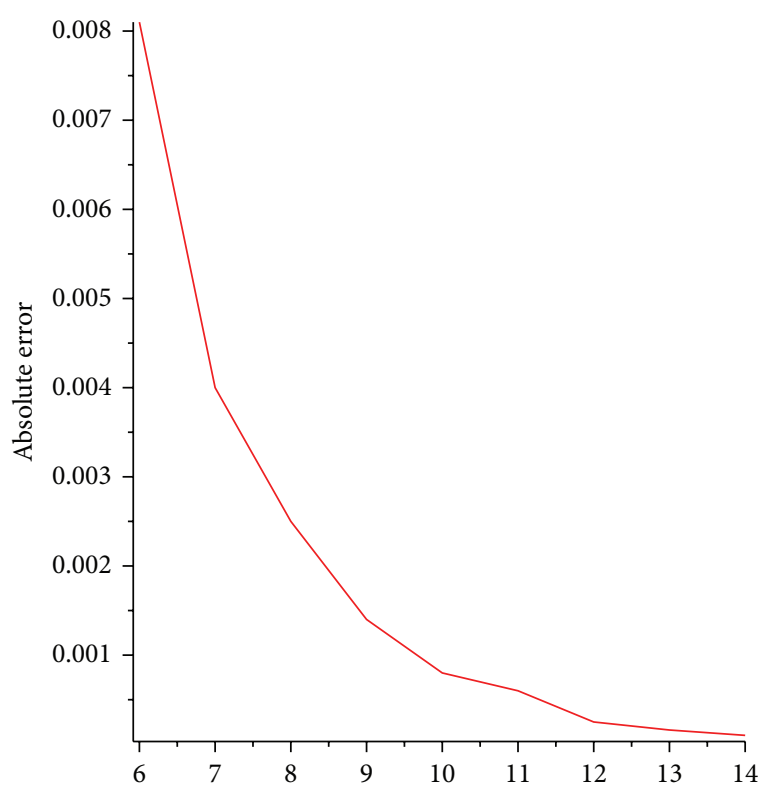

(a)

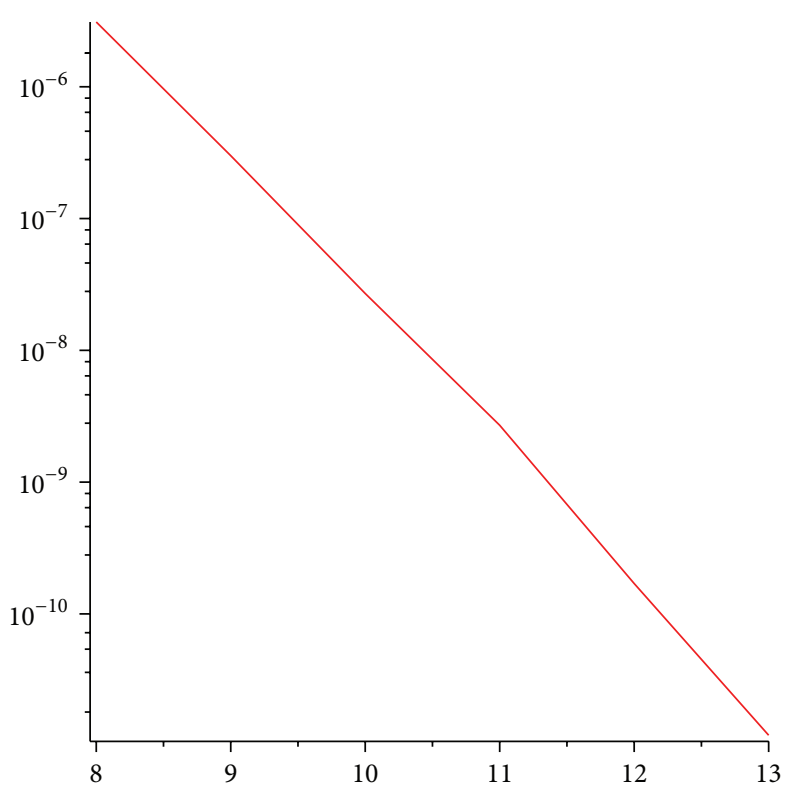

(b)

Figure 3: (a) Example 4: Horizontal axis is related number of iteration ( $n$ ) and vertical axis shows absolute error. (b) Example 5: Horizontal axis is related number of iteration $(n)$ and vertical axis shows absolute error in mod logarithmic.

TABLE 6: Comparison of the numerical result of Example 5 by present method ( $n=14$ and $m=40)$, ADM [3], and exact solution.

\begin{tabular}{lccccc}
\hline$x$ & SADM & ADM [3] & Exact & Absolute error (SADM) & Absolute error (ADM) \\
\hline 0.1 & 1.01005017 & 1.01005017 & 1.01005017 & $1.00000000 e-24$ & 0 \\
0.2 & 1.04081077 & 1.04081078 & 1.04081077 & $9.00000000 e-24$ & $1 e-9$ \\
0.3 & 1.09417428 & 1.09417428 & 1.09417428 & $7.92100000 e-20$ & $1 e-9$ \\
0.4 & 1.17351087 & 1.17351085 & 1.17351087 & $4.46670780 e-17$ & $2.3 e-8$ \\
0.5 & 1.28402542 & 1.28402507 & 1.28402542 & $6.09975969 e-15$ & $3.5 e-7$ \\
0.6 & 1.43332941 & 1.43332623 & 1.43332941 & $3.39913478 e-13$ & $3.2 e-6$ \\
0.7 & 1.63231622 & 1.63229556 & 1.63231622 & $1.02105064 e-11$ & $2.1 e-5$ \\
0.8 & 1.89648088 & 1.89637596 & 1.89648088 & $1.95219231 e-10$ & $1.1 e-4$ \\
\hline
\end{tabular}

$+(1 / 3.9 !) q^{6}\left(339 q^{2}+280\right) x^{8}+(1 / 5.11 !) q^{7}\left(1425 q^{4}+11436 q^{2}+\right.$ 4256) $x^{10}+O(11)$, where $q^{2}=1-C$. This example is solved by the SADM with $n=12$ and $m=40$ for $C=0.2,0.4$, 0.6 , and 0.8 , for which the results are shown in Tables $7-10$, respectively, and they are compared with ADM [3].

\section{Conclusion}

In this paper a new modification of the Adomian decomposition method was presented for solving Lane-Emden equations. This method, named spectral Adomian decomposition method (SADM), was successfully applied to solve the LaneEmden equations.

It is clear that in computation of integral in the right hand of (12), two difficulties may arise:

(i) In calculation, the Adomian polynomials $A_{n}(x)$ may be so much problematic that the integration becomes very complicated. (ii) By increasing $n$, the number of terms of approximate solution may increase so much rapidly that the integration becomes both complicated and timeconsuming.

To overcome the abovementioned difficulties, SADM is proposed, a combination of Adomian decomposition method and Chebyshev pseudo-spectral method. By analyzing and comparing the procedures used in SADM and ADM, it is observed that the new approach overcomes the difficulties (i) and (ii), leading to complicated calculations and timeconsuming integrals and terms not necessary in the standard ADM. Moreover, unlike spectral methods, the proposed method does not require the solution of any linear or nonlinear system of equations.

A comparative study between the proposed method and both 4th order Runge-Kutta method (RK4) and ADM was presented in tables. Consequently, the obtained results showed that SADM can solve the problem effectively, while comparison demonstrates that the proposed technique is in 
TABLE 7: The numerical results for Example 6 for $C=0.2, n=12$, and $m=40$.

\begin{tabular}{lcccc}
\hline$x$ & ADM [3] & SADM & RK4 & Residual error \\
\hline 0.1 & 0.99880903 & 0.99880903 & 0.99880903 & $4.3 e-24$ \\
0.2 & 0.99525519 & 0.99525519 & 0.99525519 & $4.3 e-24$ \\
0.3 & 0.98939500 & 0.98939500 & 0.98939500 & $4.274 e-24$ \\
0.4 & 0.98132029 & 0.98132028 & 0.98132028 & $4.211494 e-19$ \\
0.5 & 0.97115486 & 0.97115480 & 0.97115479 & $8.80749554 e-17$ \\
0.6 & 0.95905026 & 0.95904988 & 0.95904987 & $6.8967875 e-15$ \\
0.7 & 0.94518104 & 0.94517924 & 0.94517923 & $2.74001640 e-13$ \\
0.8 & 0.92574024 & 0.92973345 & 0.92973343 & $6.62093012 e-12$ \\
0.9 & 0.91293603 & 0.91291419 & 0.91291416 & $1.09390380 e-10$ \\
1 & 0.89499072 & 0.89492872 & 0.89492869 & $1.33863329 e-9$ \\
\hline
\end{tabular}

TABle 8: The numerical results for Example 6 for $C=0.4, n=12$, and $m=40$.

\begin{tabular}{lcccc}
\hline$x$ & ADM [3] & SADM & RK4 & Residual error \\
\hline 0.1 & 0.99922630 & 0.99922630 & 0.99922630 & $1.6 e-24$ \\
0.2 & 0.99691595 & 0.99691595 & 0.99691595 & $1 e-24$ \\
0.3 & 0.99310085 & 0.99310085 & 0.99310085 & $2.06 e-23$ \\
0.4 & 0.98783306 & 0.98783306 & 0.98783306 & $2.14577 e-20$ \\
0.5 & 0.98118327 & 0.98118325 & 0.98118324 & $4.49850230 e-18$ \\
0.6 & 0.97323871 & 0.97323859 & 0.97323858 & $3.53293647 e-16$ \\
0.7 & 0.96410081 & 0.96410027 & 0.96410026 & $1.40829431 e-14$ \\
0.8 & 0.95388278 & 0.95388075 & 0.95388074 & $3.41576049 e-13$ \\
0.9 & 0.94270739 & 0.94270085 & 0.94270083 & $5.66666257 e-12$ \\
1 & 0.93070541 & 0.93068681 & 0.93068679 & $6.96500076 e-11$ \\
\hline
\end{tabular}

TABLE 9: The numerical results for Example 6 for $C=0.6, n=12$, and $m=40$.

\begin{tabular}{lcccc}
\hline$x$ & ADM [3] & SADM & RK4 & Residual error \\
\hline 0.1 & 0.99957876 & 0.99957876 & 0.99957876 & $2.5 e-24$ \\
0.2 & 0.99831983 & 0.99831983 & 0.99831983 & $2 e-24$ \\
0.3 & 0.99623743 & 0.99623743 & 0.99623743 & $1 e-25$ \\
0.4 & 0.99335491 & 0.99335491 & 0.99335491 & $3.55 e-22$ \\
0.5 & 0.98970415 & 0.98970415 & 0.98970415 & $7.53053000 e-20$ \\
0.6 & 0.98532490 & 0.98532488 & 0.98532487 & $5.93212650 e-18$ \\
0.7 & 0.98026385 & 0.98026375 & 0.98026374 & $2.37324422 e-16$ \\
0.8 & 0.97457371 & 0.97457333 & 0.97457332 & $5.77974170 e-15$ \\
0.9 & 0.96831224 & 0.96831099 & 0.96831098 & $9.63164687 e-14$ \\
1 & 0.96154132 & 0.96153777 & 0.96153776 & $1.18962111 e-12$ \\
\hline
\end{tabular}

TABLE 10: The numerical results for Example 6 for $C=0.8, n=12$, and $m=40$.

\begin{tabular}{lcccc}
\hline$x$ & ADM [3] & SADM & RK4 & Residual error \\
\hline 0.1 & 0.99985103 & 0.99985103 & 0.99985103 & $3 e-25$ \\
0.2 & 0.99940531 & 0.99940531 & 0.99940531 & $5 e-26$ \\
0.3 & 0.99866642 & 0.99866642 & 0.99866642 & $8 e-26$ \\
0.4 & 0.99764024 & 0.99764024 & 0.99764025 & $1.5 e-25$ \\
0.5 & 0.99633490 & 0.99633490 & 0.99633489 & $9.087 e-23$ \\
0.6 & 0.99476060 & 0.99476060 & 0.99476059 & $7.17882 e-21$ \\
0.7 & 0.99292949 & 0.99292949 & 0.99292948 & $2.88373290 e-19$ \\
0.8 & 0.99085553 & 0.99085550 & 0.99085549 & $7.05570562 e-18$ \\
0.9 & 0.98855418 & 0.98855410 & 0.98855408 & $1.18188796 e-16$ \\
1 & 0.98604231 & 0.98604208 & 0.98604207 & $1.48604049 e-15$ \\
\hline
\end{tabular}


good agreement with the numerical results obtained using RK4.

\section{Conflict of Interests}

The authors declare that there is no conflict of interests regarding the publication of this paper.

\section{References}

[1] O. W. Richardson, The Emission of Electricity from Hot Bodies, Longmans Green and Co., London, UK, 1921.

[2] A.-M. Wazwaz, "The variational iteration method for solving nonlinear singular boundary value problems arising in various physical models," Communications in Nonlinear Science and Numerical Simulation, vol. 16, no. 10, pp. 3881-3886, 2011.

[3] A.-M. Wazwaz, "A new algorithm for solving differential equations of Lane-Emden type," Applied Mathematics and Computation, vol. 118, no. 2-3, pp. 287-310, 2001.

[4] A.-M. Wazwaz, "A new method for solving singular initial value problems in the second-order ordinary differential equations," Applied Mathematics and Computation, vol. 128, no. 1, pp. 45-57, 2002.

[5] M. I. Nouh, "Accelerated power series solution of polytropic and isothermal gas spheres," New Astronomy, vol. 9, no. 6, pp. 467473, 2004.

[6] H. Goenner and P. Havas, "Exact solutions of the generalized Lane-Emden equation," Journal of Mathematical Physics, vol. 41, no. 10, pp. 7029-7042, 2000.

[7] S. Liao, "A new analytic algorithm of Lane-Emden type equations," Applied Mathematics and Computation, vol. 142, no. 1, pp. $1-16,2003$.

[8] J.-H. He, "Variational approach to the Lane-Emden equation," Applied Mathematics and Computation, vol. 143, no. 2-3, pp. 539-541, 2003.

[9] S. A. Yousefi, "Legendre wavelets method for solving differential equations of Lane-Emden type," Applied Mathematics and Computation, vol. 181, no. 2, pp. 1417-1422, 2006.

[10] E. Momoniat and C. Harley, "Approximate implicit solution of a Lane-Emden equation," New Astronomy, vol. 11, no. 7, pp. 520526, 2006.

[11] A. Yildirim and T. Öziş, "Solutions of singular IVPs of LaneEmden type by homotopy perturbation method," Physics Letters, Section A: General, Atomic and Solid State Physics, vol. 369, no. 1-2, pp. 70-76, 2007.

[12] M. S. H. Chowdhury and I. Hashim, "Solutions of a class of singular second-order IVPs by homotopy-perturbation method," Physics Letters A, vol. 365, no. 5-6, pp. 439-447, 2007.

[13] J. I. Ramos, "Series approach to the Lane-Emden equation and comparison with the homotopy perturbation method," Chaos, Solitons \& Fractals, vol. 38, no. 2, pp. 400-408, 2008.

[14] C. M. Khalique and P. Ntsime, "Exact solutions of the LaneEmden-type equation," New Astronomy, vol. 13, no. 7, pp. 476480, 2008.

[15] O. P. Singh, R. K. Pandey, and V. K. Singh, "An analytic algorithm of Lane-Emden type equations arising in astrophysics using modified homotopy analysis method," Computer Physics Communications, vol. 180, no. 7, pp. 1116-1124, 2009.

[16] K. Parand, M. Shahini, and M. Dehghan, "Rational Legendre pseudospectral approach for solving nonlinear differential equations of LANe-Emden type," Journal of Computational Physics, vol. 228, no. 23, pp. 8830-8840, 2009.

[17] A. Yıldırım and T. Öziş, "Solutions of singular IVPs of LaneEmden type by the variational iteration method," Nonlinear Analysis: Theory, Methods \& Applications, vol. 70, no. 6, pp. 2480-2484, 2009.

[18] S. Iqbal and A. Javed, "Application of optimal homotopy asymptotic method for the analytic solution of singular LaneEmden type equation," Applied Mathematics and Computation, vol. 217, no. 19, pp. 7753-7761, 2011.

[19] R. K. Pandey and N. Kumar, "Solution of Lane-Emden type equations using Bernstein operational matrix of differentiation," New Astronomy, vol. 17, no. 3, pp. 303-308, 2012.

[20] A. H. Bhrawy and A. S. Alofi, "A Jacobi-Gauss collocation method for solving nonlinear Lane-Emden type equations," Communications in Nonlinear Science and Numerical Simulation, vol. 17, no. 1, pp. 62-70, 2012.

[21] M. Heydari, S. M. Hosseini, and G. B. Loghmani, "Numerical solutions of singular IVPs of lane emden type using integral operator and radial basis functions," International Journal of Industrial Mathematics, vol. 4, no. 2, 12 pages, 2012.

[22] E. H. Doha, W. M. Abd- Elhameed, and Y. H. Youssri, "Second kind Chebyshev operational matrix algorithm for solving differential equations of Lane-Emden type," New Astronomy, vol. 23-24, pp. 113-117, 2013.

[23] A. Nazari-Golshan, S. S. Nourazar, H. Ghafoori-Fard, A. Yildirim, and A. Campo, "A modified homotopy perturbation method coupled with the Fourier transform for nonlinear and singular Lane-Emden equations," Applied Mathematics Letters, vol. 26, no. 10, pp. 1018-1025, 2013.

[24] G. Adomian, Nonlinear Stochastic Operator Equations, Academic Press, New York, NY, USA, 1986.

[25] S. Abbasbandy, "A numerical solution of Blasius equation by Adomian's decomposition method and comparison with homotopy perturbation method," Chaos, Solitons \& Fractals, vol. 31, no. 1, pp. 257-260, 2007.

[26] A.-M. Wazwaz, A First Course in Integral Equations, World Scientific, 1997.

[27] I. L. El-Kalla, "Convergence of the Adomian method applied to a class of nonlinear integral equations," Applied Mathematics Letters, vol. 21, no. 4, pp. 372-376, 2008.

[28] C. Canuto, M. Y. Hussaini, A. Quarteroni, and T. A. Zang, Spectral Methods in Fluid Dynamics, Springer, New York, NY, USA, 1988. 


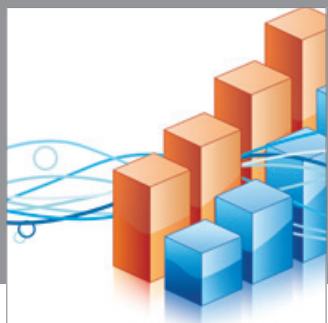

Advances in

Operations Research

mansans

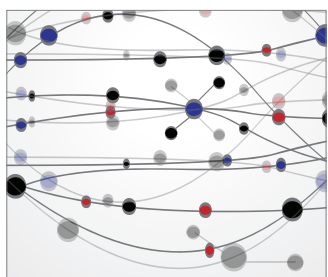

The Scientific World Journal
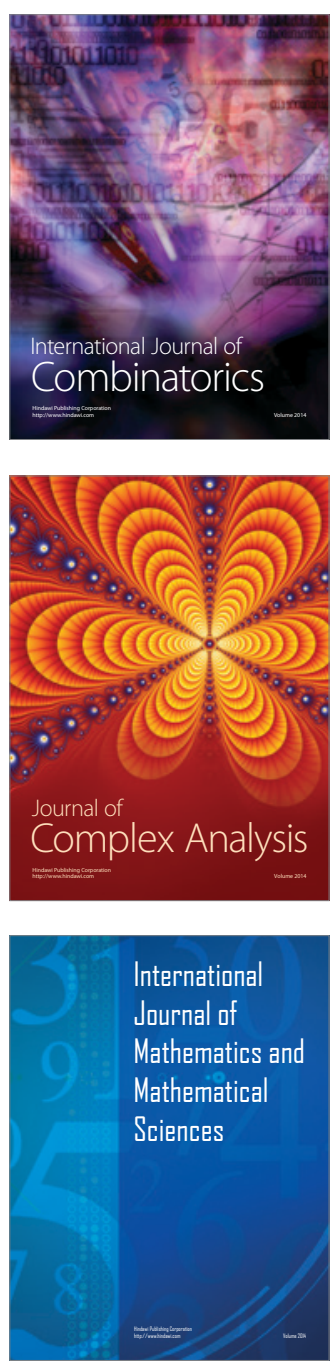
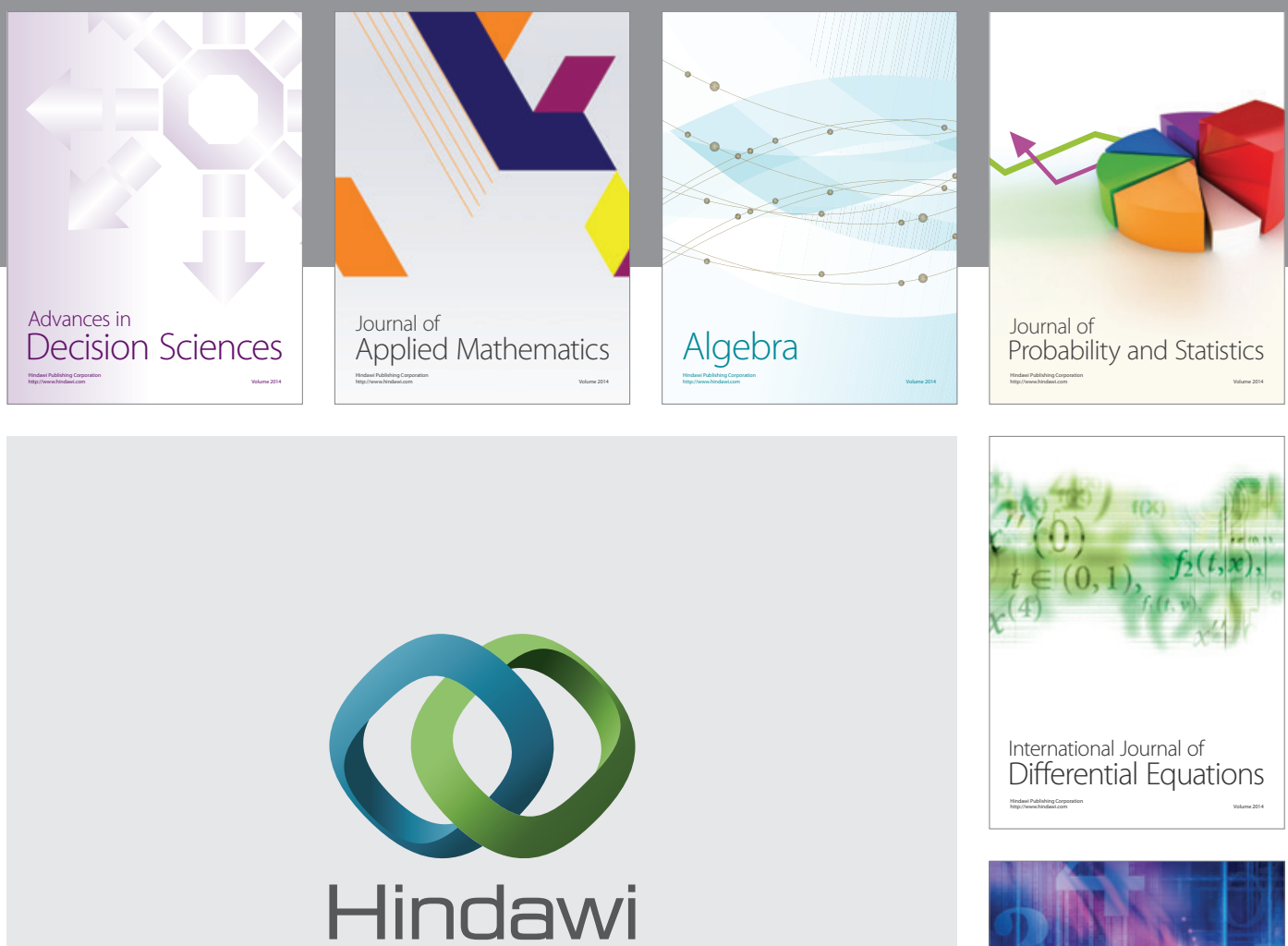

Submit your manuscripts at http://www.hindawi.com
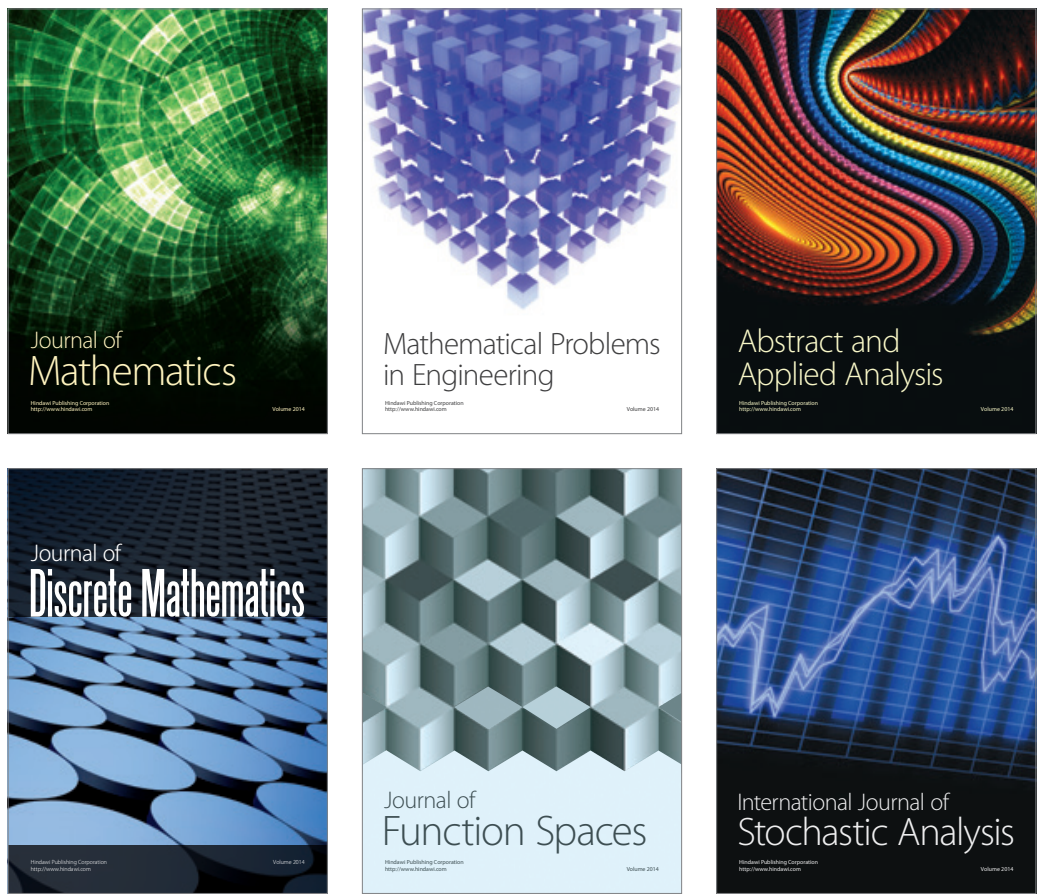

Journal of

Function Spaces

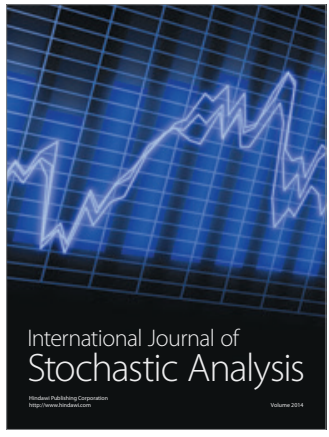

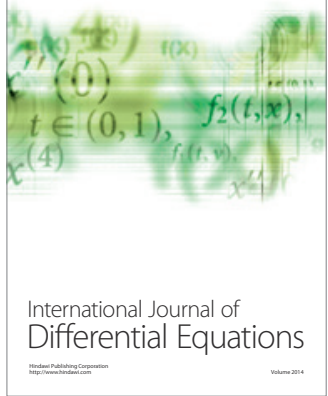
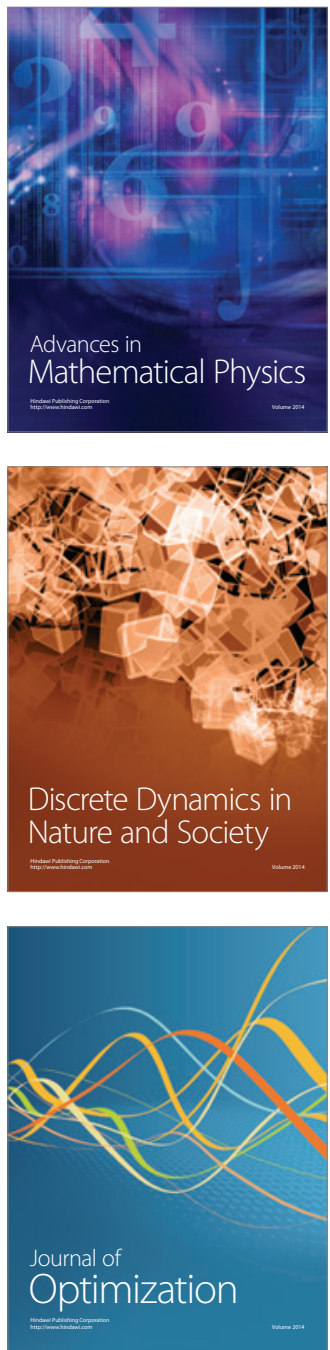